\section{Virus Ecology: Studying Animal Reservoirs to Understand Viral Emergence}

\section{Drosten ${ }^{1}$}

${ }^{1}$ Institute of Virology, University of Bonn, Germany

Ten years after the SARS epidemic, zoonotic and emerging viruses have become a growing field of research. Some remarkable novel virus descriptions in animals have demonstrated how ignorant we are of the diversity of viruses around us. In our efforts to delineate viral origins we may have to re-assess our concept of reservoir. In many instances, we are mixing up ecological and epidemiological implications of viral evolution. Among the biggest challenges in this field is the integration of the concepts of virus-host codivergence, and viral host switching. In addition, assessments of viral reservoirs with the intention to predict future pandemic threats would have to take into account important host and virus traits which cannot be predicted merely from virus genes. For example, we need to know whether there are hosts which have a high- er propensity to carry broader spectra or higher concentrations of viruses, potentially without being affected. Among the viruses borne in such reservoirs, there may be some that are more promiscuous in their choice of hosts than others, potentially due to the conservedness of their receptor structures or the way they interfere with conserved- or not-soconserved immune properties. A synopsis of available approaches demonstrates how much work needs to be done before we will be able to assess functional, rather than genetic diversity of reservoir-borne viruses.

\section{Conflicts of Interest: none}

Bibliography

DOI http://dx.doi.org/10.1055/s-0033-1358040

Drug Res 2014; 64, Suppl. 1: S27-S27

(c) Georg Thieme Verlag KG Stuttgart · New York . ISSN 2194-9379

\section{Correspondence}

\section{Christian Drosten}

Universitätsklinikum Bonn

Sigmund-Freud-Straße 25

53105 Bonn

drosten@virology-bonn.de 